\title{
Substituting model based indicators in Harvest Control Rules by observations using fuzzy logic methodology
}

\author{
Arne Eide \\ UiT - The Arctic University of Norway, \\ The Norwegian College of Fishery Science, Tromsø, Norway \\ arne.eide@uit.no
}

\begin{abstract}
Harvest Control Rules are predefined heuristic decision rules to provide quota advices for managed fisheries. Frequently statistical methods and biological assumptions expressed in mathematical models, are used to provide the Harvest Control Rules with initial information (indicators values). The aim of this paper is to investigate a possible way forward of replacing these inputs by quantities of measurable observations, e.g. catch-at-age statistics. The paper presents a method by which recruitment indexes and stock biomass indicators are obtained by non-parametric use of annual catch-at-age records, without filtering the raw data (observations) through mathematical models. Two related methods, applied on three empirical cases, are provided: First, showing that recruitment strengths of the Northeast Arctic cod, haddock and saithe stocks, obtained by fuzzy logic methodology, are satisfactory captures by the use of catch-at-age data. Secondly, stock size indicators are estimated for the three species by the same catch-at-age data. The second task turns out to be more challenging than the first, but also in the case of stock size evaluation, the suggested procedure provides reasonable results when compared to standard stock assessment methods.
\end{abstract}

Keywords: Stock assessment; Fuzzy logic; Northeast Arctic fisheries; and Harvest Control Rules 


\section{Introduction}

Following the principles of the 1992 UN Rio Declaration, FAO in 1996 issued guidelines on precautionary approach to fisheries and species introduction (Anon., 1996; Kvamsdal et al., 2016). In 2000 the Convention on Biological Diversity led to the identification of Ecosystem Approach as an overall strategy for the management of natural resources. Anon. (2006) provides a detailed description on how to implement the principles in fisheries within the area of the International Council for the Exploration of the Sea (ICES). A large number of fishing nations and fishery management institutions have adopted the guidelines (Punt, 2006) and Harvest Control Rules (HCRs) have become a methodology of precautionary management in many fisheries.

Up to recently there has been a widespread belief among fishery management institutions that all knowledge necessary for sound management of fisheries could be embedded in biological models, capturing the dynamics of biological systems as well as its interactions with fishing activities. Management failures, and the broader ecosystem perspective, have created scepticism towards the use of mathematical modelling in fisheries management. Schnute and Richards (2001) ask managers to be sceptical, to expand their knowledge base, and to apply common sense when using models. In the development of fisheries management we may have arrived at a point where it is fair to ask: Is it possible to obtain sufficient scientific insight to build ecosystem models suitable for management? What will it cost, and can it be done in a transparent way? Reduced faith in the modelling approach, combined with the expenses associated with feeding increasingly data demanding models (Degnbol, 2001), call for new approaches to management and more efficient use of existing system knowledge, which not necessarily could be implemented in the model formulations. Increasingly complex and data demanding models lead to reduced transparency, reduced legitimacy, and reduced belief in the modelling approach to management (Dankel et al., 2016; Kelly and Codling, 2006).

Stock assessment methods based on mathematical modelling and statistical techniques have undoubtedly been crucial for today's level of understanding of fisheries dynamics and for the successful management of many fisheries. This achievement makes it is also easier to express and utilise heuristic HCR procedures in fisheries management. It is fair to say that HCRs have been introduced as a continuation of previous management procedures based on mathematical models. This may also turn out to be a problem for a further utilisation of the capacities of a fully developed heuristic expert system (Clancey, 1983) for fisheries management.

Today HCRs are successfully introduced in a large number of fisheries (Kvamsdal et al., 2016). In principle HCRs should not limited to only include biological measures (Garcia et al., 2009). Rule based procedures open for the use non-biological indicators (such as social and economic measures), possibilities that so far are poorly explored and 
hardly utilised. Heuristic decision rules (expert systems) allow vague knowledge and unspecified uncertainties to be integrated in the formulation of rules. Hypotheses and/or vague knowledge believed to be important (from biological, economic or societal points of view), can be added to existing sets of rules by reformulating existing rules or adding new. Reservation levels and intervals between 'go' and 'no' zones may include uncertainty and vagueness in the evaluation of tolerance levels. Together it constitutes the precautionary evaluation of possible short and long term consequences different levels of fish harvest may have on other parts of the ecosystem, the economy, and the society as a whole.

Heuristic decision rules organised in sets of HCRs, are used to evaluate the state of fisheries. The goal of the evaluation is not to identify stock size or fishing pressure but to estimate membership degrees in fuzzy sets, such as for example in the sets of healthy stocks and overexploited stocks illustrated by Figure 1. Being a fuzzy logic expert system, HCRs use membership functions, as those indicated in the figure, to identify the sets of highest membership degrees. This information is then used to decide the corresponding advice on TAC (Total Allowable Catch), all based on pre-defined rules. Crisp value estimations of stock size or other stock properties are merely means by which the selection of dominating fuzzy sets can be made.
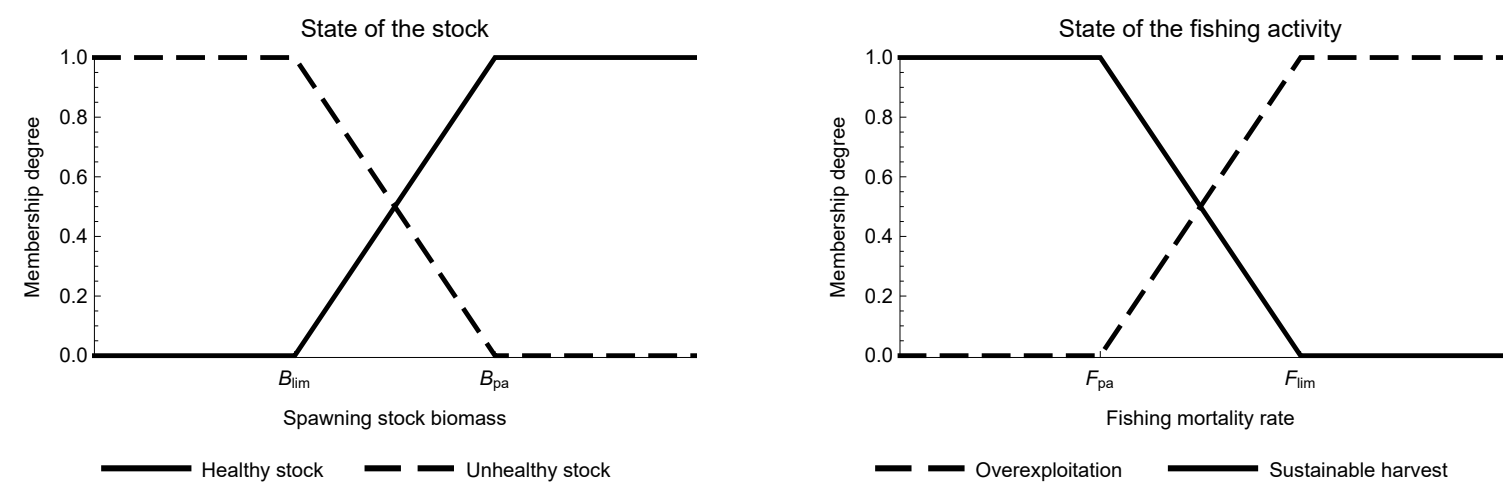

Figure 1: Standard membership functions used in the simplest versions of Harvest Control Rules. Membership degrees in four fuzzy sets are determined by the value of two indicators, which are measured along the two horizontal axes. $B_{l i m}$ is the limit value of spawning biomass below which the stock is $100 \%$ unhealthy, while the stock is $100 \%$ healthy aboe the precautionary level at $B_{p a}$. The corresponding values to the right is the critically high fishing mortality rate $F_{\text {lim }}$ above which $100 \%$ overfishing occurs, while a $100 \%$ sustainable fishery takes place at and below the precautionary value $F_{p a}$.

Up to now HCRs in fisheries management have been implemented as an extension of previous management models. Indicators have been built on previous reference points and identified within the modelling framework developed prior to the HCRs. The rules commonly use model estimates of spawning biomasses and fishing mortality rates as indicators (inputs to the HCRs), as indicated in Figure 1. Hence, the transition from a model based to a rule based control system has been made possible, but is not yet completed. Since model outputs are not the goal, but rather a mean in the management 
procedure, it is fair to ask: Could the parametric modelling part of the procedure be substituted by more direct use of basic observations and expert knowledge?

There are several reasons why the use of modelled indicator values in HCRs is problematic. Inclusion of model uncertainty (Dankel et al., 2016) and lack of transparency (Kelly and Codling, 2006) are often mentioned as the most serious problems. There is also a tautological problem of model based inputs when targets and limits (illustrated by $p a$ - and lim-levels in Figure 1) are identified by using similar assumptions as those embedded in the model. The example in Figure 1 shows how estimated values of spawning stock biomass and fishing mortality rate - commonly used as indicators in HCRs link to membership degrees of different fuzzy sets. Indicator values are often interrelated; estimated within the same modelling framework and sharing observation and modelling uncertainties.

Feigenbaum (1977) sets up a road map for heuristic rule-based case studies where the central organising principle is what he refers to as the Line-of-reasoning, which should be possible to explain in a language convenient to the user. Feigenbaum claims this to be necessary for the right use of an expert system (which in his terminology is called knowledge engineering), and for system development (debugging and extending the knowledge base). It may be questioned if a comprehensible Line-of-reasoning today exists for the users of management advices.

The aim of this paper is to investigate a possible approach of omitting the use of parametric models without losing important information hidden in data set structures; information which today is filtered through model assumptions and non-transparent tuning procedures. The Virtual Population Analysis (VPA; see Gulland, 1965) is an example of a fairly transparent accounting system based on consistency and fixed natural mortality rates. The less transparent eXtended Survivor Analysis (XSA) with improved tuning procedures, employing information from other data sources, clearly provides more reliable estimates of recent stock history (Shepherd, 1999). For the case studies provided in this paper - the Northeast Arctic (NEA) cod, haddock and saithe fisheries - existing XSA estimates are compared with results from suggested alternative methods, acknowledging XSA as a model within the class of well functioning integrated statistical year class models.

\section{Data and method}

A simple method on how to utilise fuzzy set theory on catch-at-age data for the evaluation of year class (or recruitment) strength in age structured fish populations is presented below, providing examples from the NEA cod, haddock and saithe stocks. The methodology builds to some extent on the ideas of Sakuramoto (1995) and Mackinson et al. (1999), also referred to in Eide (2016) where a number of management performance indicators 
are suggested. Sakuramoto (1995) and Mackinson et al. (1999) show how catch-at-age data can be used to estimate recruitment strength by the use of fuzzy logic techniques.

Table 1: Registered catch quantities in thousand tons distributed on year classes (year of birth) and age of capture (from 3 to 13 and above), from tables 3.6 and 3.8 in Anon. $(2016)$.

\begin{tabular}{|c|c|c|c|c|c|c|c|c|c|c|c|}
\hline Year class & 3 & 4 & 5 & 6 & 7 & 8 & 9 & 10 & 11 & 12 & $13+$ \\
\hline 1944 & 0.227 & 2.052 & 39.088 & 76.896 & 95.339 & 76.285 & 53.429 & 57.760 & 29.808 & 13.819 & 8.690 \\
\hline 1945 & 0.048 & 4.561 & 37.468 & 88.110 & 130.387 & 62.730 & 63.671 & 66.746 & 31.616 & 11.285 & 5.036 \\
\hline 1946 & 0.367 & 7.011 & 88.978 & 141.748 & 63.905 & 46.387 & 67.194 & 54.345 & 26.632 & 10.251 & 5.961 \\
\hline 1948 & 9.875 & 96.563 & 143.411 & 198.479 & 231.085 & 217.137 & 97.431 & 76.991 & 26.873 & 15.297 & 15.511 \\
\hline 1949 & 10.604 & 81.821 & 184.458 & 279.111 & 250.806 & 167.832 & 74.826 & 61.071 & 35.086 & 16.147 & 9.858 \\
\hline 1950 & 18.965 & 119.482 & 228.072 & 458.364 & 251.525 & 150.659 & 125.993 & 99.121 & 48.261 & 17.392 & 9.988 \\
\hline 1951 & 5.048 & 21.462 & 138.889 & 128.678 & 112.837 & 76.615 & 44.309 & 55.241 & 24.130 & 11.593 & 10.080 \\
\hline 1955 & 10.614 & 56.118 & 70.002 & 112.567 & 85.740 & 80.456 & 39.716 & 11.061 & 3.279 & 3.346 & 3.564 \\
\hline 1956 & 11.308 & 49.911 & 129.631 & 152.082 & 107.975 & 45.535 & 20.450 & 10.869 & 6.204 & 1.179 & 2.700 \\
\hline 1957 & 12.880 & 72.960 & 155.534 & 165.212 & 63.837 & 29.702 & 27.213 & 16.559 & 5.920 & 4.434 & 2.533 \\
\hline 1958 & 14.098 & 93.811 & 197.327 & 108.950 & 61.320 & 70.670 & 55.369 & 24.319 & 15.935 & 2.857 & 2.797 \\
\hline 1959 & 13.573 & 65.260 & 93.052 & 102.081 & 91.436 & 87.484 & 61.116 & 32.742 & 11.051 & 4.527 & 6.507 \\
\hline 1960 & 4.223 & 25.252 & 80.648 & 75.719 & 73.889 & 69.040 & 67.584 & 24.692 & 6.568 & 4.268 & 2.711 \\
\hline 1961 & 1.748 & 17.679 & 40.918 & 45.004 & 83.841 & 102.828 & 49.935 & 17.354 & 11.567 & 2.266 & 3.919 \\
\hline 1967 & 2.651 & 12.090 & 38.370 & 46.486 & 40.542 & 40.873 & 23.820 & 13.126 & 9.267 & 1.122 & 0.838 \\
\hline 1968 & 3.489 & 34.982 & 93.940 & 104.353 & 87.090 & 58.920 & 36.557 & 9.791 & 3.937 & 0.733 & 0.571 \\
\hline 1969 & 13.504 & 119.659 & 238.413 & 225.277 & 132.127 & 106.421 & 61.528 & 15.369 & 5.994 & 1.174 & 0.519 \\
\hline 1970 & 111.820 & 288.669 & 251.577 & 237.654 & 204.009 & 141.922 & 72.209 & 23.203 & 9.543 & 2.803 & 1.413 \\
\hline 1971 & 29.394 & 38.271 & 95.192 & 108.496 & 77.237 & 35.495 & 20.487 & 12.009 & 3.317 & 1.199 & 0.594 \\
\hline 1972 & 18.566 & 83.469 & 194.959 & 122.169 & 52.967 & 31.256 & 28.034 & 6.025 & 2.645 & 1.311 & 0.995 \\
\hline 1973 & 29.868 & 151.748 & 128.318 & 68.358 & 53.345 & 48.175 & 18.592 & 6.001 & 1.886 & 0.510 & 0.916 \\
\hline 1974 & 19.401 & 36.774 & 54.159 & 68.905 & 64.928 & 35.595 & 19.775 & 5.794 & 1.617 & 1.457 & 1.338 \\
\hline 1975 & 38.623 & 54.239 & 83.626 & 132.575 & 124.513 & 102.276 & 36.048 & 7.534 & 5.464 & 3.114 & 1.036 \\
\hline 1976 & 3.010 & 9.568 & 29.956 & 55.887 & 67.846 & 40.689 & 11.927 & 5.583 & 2.276 & 0.972 & 0.461 \\
\hline 1977 & 1.056 & 9.277 & 26.116 & 50.496 & 59.217 & 22.632 & 6.666 & 4.368 & 1.204 & 0.149 & 0.151 \\
\hline 1978 & 1.669 & 13.816 & 42.789 & 63.070 & 52.638 & 23.728 & 7.826 & 4.936 & 0.454 & 0.253 & 0.067 \\
\hline 1979 & 3.311 & 26.844 & 46.829 & 62.526 & 52.582 & 22.887 & 9.962 & 2.645 & 0.782 & 0.199 & 0.186 \\
\hline 1980 & 2.611 & 27.483 & 56.168 & 70.350 & 47.152 & 17.676 & 6.219 & 1.761 & 0.566 & 0.946 & 1.219 \\
\hline 1981 & 9.858 & 62.704 & 147.903 & 143.078 & 73.973 & 25.525 & 6.044 & 3.775 & 2.672 & 3.811 & 1.725 \\
\hline 1992 & 3.757 & 31.948 & 106.538 & 93.134 & 50.647 & 29.913 & 10.709 & 4.052 & 1.521 & 1.527 & 0.750 \\
\hline 1993 & 6.047 & 37.951 & 79.335 & 72.865 & 42.321 & 25.171 & 11.166 & 4.034 & 2.711 & 1.150 & 2.294 \\
\hline 1994 & 8.594 & 93.318 & 142.937 & 110.471 & 80.007 & 55.099 & 18.361 & 13.933 & 5.463 & 1.802 & 1.229 \\
\hline 1995 & 21.683 & 78.491 & 149.801 & 156.765 & 148.269 & 70.570 & 41.864 & 19.230 & 5.675 & 2.898 & 1.107 \\
\hline 1996 & 4.726 & 34.418 & 100.665 & 156.285 & 131.407 & 85.517 & 39.156 & 12.123 & 4.515 & 2.317 & 1.406 \\
\hline 1997 & 2.680 & 36.770 & 118.833 & 177.773 & 166.240 & 91.255 & 39.630 & 11.158 & 6.006 & 8.439 & 3.107 \\
\hline 1998 & 3.329 & 35.067 & 117.938 & 166.091 & 145.973 & 71.128 & 26.846 & 13.904 & 7.560 & 8.354 & 5.914 \\
\hline 1999 & 1.691 & 23.391 & 81.830 & 136.280 & 105.476 & 56.650 & 34.229 & 15.676 & 25.880 & 6.432 & 5.844 \\
\hline 2000 & 4.866 & 43.195 & 177.636 & 186.779 & 131.377 & 105.861 & 57.356 & 31.659 & 27.741 & 13.050 & 9.104 \\
\hline 2001 & 1.505 & 21.349 & 53.800 & 66.021 & 53.200 & 45.031 & 29.900 & 37.783 & 19.934 & 7.828 & 8.582 \\
\hline 2002 & 4.012 & 54.760 & 115.065 & 98.903 & 80.246 & 85.992 & 72.355 & 42.963 & 26.904 & 11.515 & 10.392 \\
\hline 2003 & 6.155 & 53.019 & 76.027 & 112.866 & 125.110 & 97.556 & 77.407 & 66.835 & 32.226 & 22.439 & \\
\hline 2004 & 18.850 & 65.664 & 146.157 & 185.081 & 236.400 & 212.586 & 262.952 & 158.711 & 117.961 & & \\
\hline 2005 & 6.513 & 45.292 & 93.708 & 168.123 & 218.444 & 314.183 & 260.995 & 162.541 & & & \\
\hline 2006 & 3.650 & 19.432 & 55.852 & 94.470 & 172.997 & 236.219 & 132.446 & & & & \\
\hline 2007 & 1.387 & 10.523 & 28.798 & 49.607 & 98.075 & 100.484 & & & & & \\
\hline 2008 & 1.106 & 11.927 & 37.996 & 85.721 & 105.959 & & & & & & \\
\hline 2009 & 1.806 & 15.981 & 66.444 & 111.636 & & & & & & & \\
\hline 2010 & 2.061 & 23.071 & 63.831 & & & & & & & & \\
\hline 2011 & 4.135 & 34.207 & & & & & & & & & \\
\hline 2012 & 3.366 & & & & & & & & & & \\
\hline
\end{tabular}

Fuzzy set theory was first introduced by Lotfi Zadeh in the 1960ies (Zadeh, 1983 and 2002) and soon proved to be a practical tool with a wide area of applications, also related to fisheries management (Sakuramoto, 1995; Silvert, 1997; Mackinson et al., 1999). The theory extends traditional set theory by introducing the concept of degrees of set 
memberships. A fish stock could for example have partial membership in both the set of large stocks and the set of small fish stocks at the same time. Furthermore, it may be partial member in the set of overexploited stocks and the set of well-managed stocks at the same time (which in the HCR context could be when being between precautionary and limit values of a given indicator, see Figure 1). The principle provides a method to include characteristics that gradually change (e.g. age, size, etc.) or genuine vagueness in set theoretic studies. It is easy to link this to real world problems as for example to the management of fisheries. Obviously, there is no crisp value identifying the point at which a stock becomes for example overexploited. Similarly, a single fish - or a person - could have partial membership both in the set of young individuals and in the set of old individuals. As observers, however, we do not need to know the age of a person to have an opinion of in which of the two sets (of young and old individuals) the person has the highest membership degree. The phenomena is known from ancient time as the Sorites paradox. It refers to real vagueness of terms often involved in human evaluations and decisions. Fuzzy set theory aims to operationalise how to make decisions within this framework.

Table 2: Sixth part quantiles of each data set of catches-at-age from 1946 to 2015, illustrated graphically as curves in Figure 2

\begin{tabular}{c|rrrrrrrrrrr|} 
Quantiles & \multicolumn{10}{c}{ Age groups } \\
& 3 & 4 & 5 & 6 & 7 & 8 & 9 & 10 & 11 & 12 & $13+$ \\
\hline $5 / 6$ & 18.566 & 83.469 & 178.660 & 185.080 & 173.000 & 132.470 & 77.407 & 55.241 & 26.904 & 11.285 & 8.582 \\
$4 / 6$ & 8.594 & 56.118 & 137.330 & 152.080 & 124.510 & 97.556 & 57.356 & 28.673 & 11.567 & 4.839 & 3.859 \\
$3 / 6$ & 4.866 & 36.774 & 93.467 & 112.570 & 95.339 & 70.570 & 39.716 & 15.676 & 6.204 & 2.898 & 2.533 \\
$2 / 6$ & 3.329 & 21.462 & 63.831 & 93.134 & 73.973 & 48.175 & 27.382 & 11.158 & 4.287 & 1.463 & 1.219 \\
$1 / 6$ & 1.505 & 13.816 & 39.088 & 66.021 & 53.345 & 31.871 & 15.022 & 6.025 & 2.276 & 0.984 & 0.594 \\
\hline
\end{tabular}

Table 1 shows the NEA cod catch-at-age data for the year classes from 1943 to 2012, available from the ICES Arctic Fisheries Working Group (AFWG) (Anon., 2016). The tabled data is presented as a Box-Whisker-plot in Figure 2 where the range of each box is defined by the $25 \%$ and $75 \%$ quantiles of catches-at-age over the covered period. These quantiles split, together with the $50 \%$ quantile (the median value), each data set into four fractions. For the purpose of this study we prefer to split each catch-at-age data set into six fractions, obtained by employing the five $1 / 6$ quantiles (within the ranges of $16.7 \%$, $33.3 \%, 50 \%, 66.7 \%$, and $83.3 \%$ of the total catches-at-age).

Table 2 shows the $1 / 6$ quantile values within the catches of each age group. The curves in Figure 2 connect the 1/6 quantile of each data set, serving as a measure of classifying each year class by catch-at-age data in sets of very weak, weak, medium, strong and very strong year class catches. For example, catches of age group 5 less than 39.088 thousand tons (which is the $1 / 6$ quantile of age 5 years, see Table 2) is assumed to reflect a very weak year class, while catches above 178.660 thousand tons at the same age is assumed to reflect a very strong year classes. In the first case the catch is a $100 \%$ member of the set 
of very weak year classes, while catches above 178.660 thousand tons are $100 \%$ members in the set of very strong year classes.

Table 3: Year class evaluations by membership degrees of sets of year class classification (in percent) at the quantile values provided in Table 2.

\begin{tabular}{l|rrrrr} 
Catches & \multicolumn{5}{|c}{ Year class evaluation in terms of percentage membership } \\
& Very weak (set 1) & Weak (set 2) & Medium (set 3) & Strong (set 4) & Very strong (set 5) \\
\hline$\geqslant 5 / 6$ quantile & 0 & 0 & 0 & 0 & 100 \\
$=4 / 6$ quantile & 0 & 0 & 0 & 0 & 0 \\
$=3 / 6$ quantile & 0 & 0 & 100 & 0 & 0 \\
$=2 / 6$ quantile & 0 & 100 & 0 & 0 \\
$\leqslant 1 / 6$ quantile & 100 & 0 & 0 & 0 \\
\hline
\end{tabular}

Catch values laying between the $1 / 6$ and the $2 / 6$ quantiles within their respective age groups are also partial members in the set of very weak year classes, but with less than $100 \%$ memberships. The exact membership degree depends on the distances to the two quantile values. Similarly, these catches also have partial memberships in the set of weak year classes. Zadeh (1983) refers to sets in which partial membership is possible as fuzzy sets. In this study any catch-at-age may be partial or full member of one or two fuzzy sets, according to membership degrees determined by linear relationships between the quantiles defining $0 \%$ and 100\% memberships (respectively representing normalised membership degrees of 0 and 1) in each set (Table 3) as shown in Figure 3.

After the fuzzification of catches, which is to determine the membership degrees of each catch-at-age observation in the five defined sets, a defuzzification procedure is performed after the principle of Highest Membership Degree, selecting the set in which each year class' catch-at-age has its highest membership degree. By this procedure each catchat-age is associated with a single set as illustrated in the top eleven rows of Figure 4. This simplification describes the strength of each year class in terms of eleven evaluations (except in the recent cases where the year class is still present in the stock). Other simple defuzzification procedures could be chosen (centre of gravity, centre of set intersections, centre of largest integrated set, all are possible candidates). Intuitively the highest membership degree seems to be most appealing in our case, but the choice of defuzzification method is one factor that should be further analysed and discussed. However, this study will employ to the principle of Highest Membership Degree.

Assign a numerical value to each of the five sets; Set 1 is the set of very weak year classes, set 2 the set of weak year classes, set 3 the set of medium year classes, set 4 the set of strong year classes, and set 5 is the set of very strong year classes. The 1943 year class, which was three years old while recruited to the fishable NEA cod stock in 1946, is for example evaluated by catch-at-age data to have the highest membership degrees in the following sets: $\{1,1,1,4,4,5,5,5,5,5,5\}$, listed from the lowest (3 years) to the highest age (13 years and older).

The second defuzzification procedure is to convert the age-structured vector of a year class - characterising year class strength at each catch-at-age value of the year class - to 


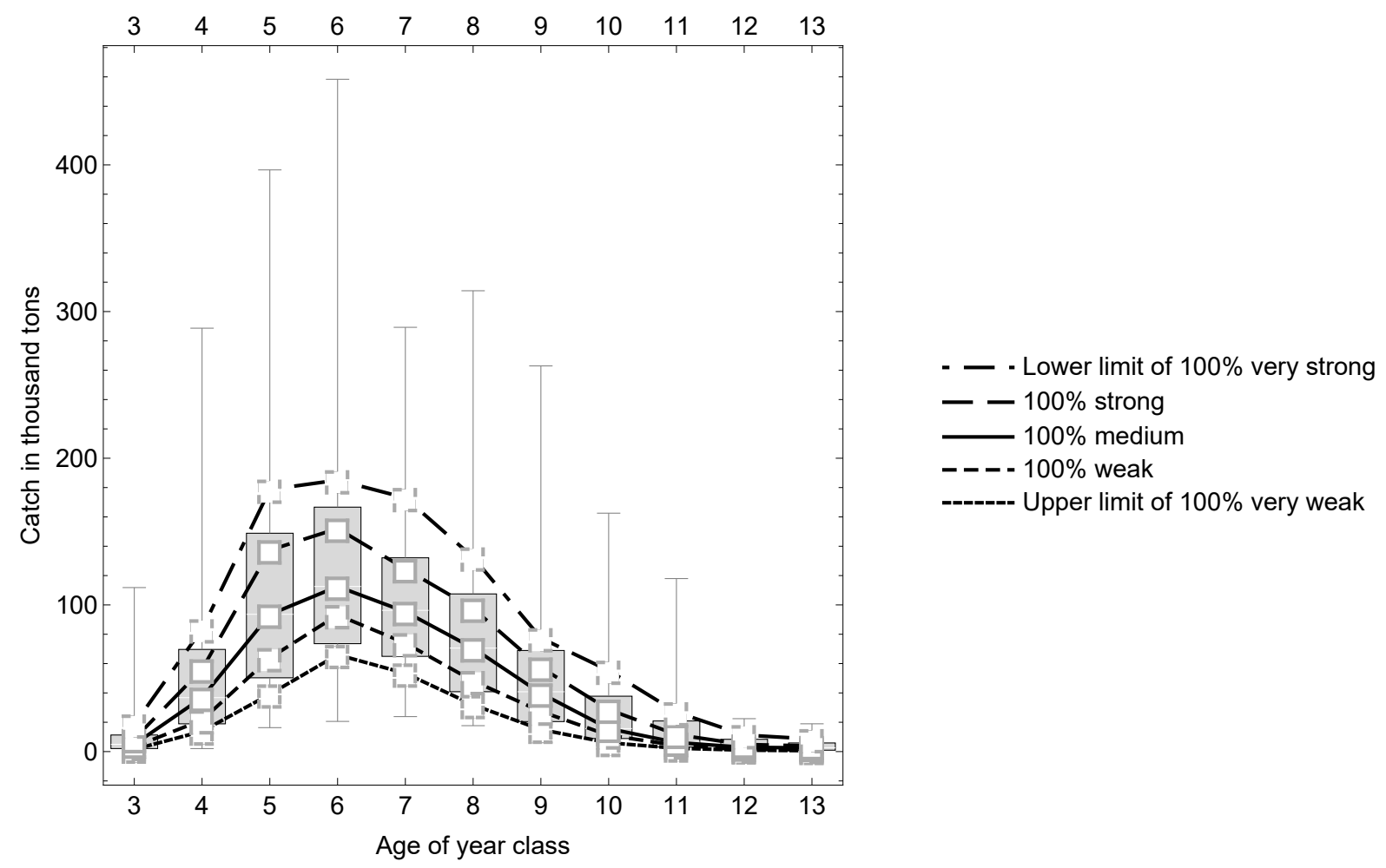

Figure 2: The box-whisker plot gives the distribution in NEA cod catches (in thousand tons) during the period 1946-2015 separated on year classes. The median values are indicated by white lines through the boxes. The five curves connect the different $1 / 6$ quantiles of each year class, quantile values (from Table 2) are marked by white squares. Data sources are table 3.6 and table 3.8 in Anon. (2016), here presented in Table 1.

one single value, being the strength evaluation for the entire year class. As in the previous defuzzication procedure, where the simple principle of Highest Membership Degree where chosen, also here numerous possible defuzzification principles are available. Simple and transparent rule-based methods are preferable, and in this case we employ a successive pairwise evaluation of the elements in the year class vector following the rules displayed in Table 4. According to Figure 2, the largest cod catches are represented at ages between 4 and 9 year; hence, it seems reasonable to place more emphasis on these catches. It may also be reasonable to put a larger emphasis on early catches of a year class because of the increasing impact previous catches will have on the catches of older fish.

The chosen procedure therefore starts by pairing the set values of the oldest and youngest age group first ( 3 and 13+), followed by the pairing of ( 3 and 4$),(4$ and 5$),(5$ and 6$),(6$ and 7$),(7$ and 8$),(8$ and 9$),(9$ and 10) and (10 and 11). The second last value (in this case the catches of 12 year old cod) is omitted, systematically emphasising the catches on younger fish. The pairwise evaluation results in a new vector where the number of elements is reduced from 11 to 9 : $\{3,1,1,2,4,5,5,5,5\}$. Then the same procedure is repeated, applying the same rules and the same pairing principle, until the vector contains only one digit. In our example, the sequence of vectors continues in this 

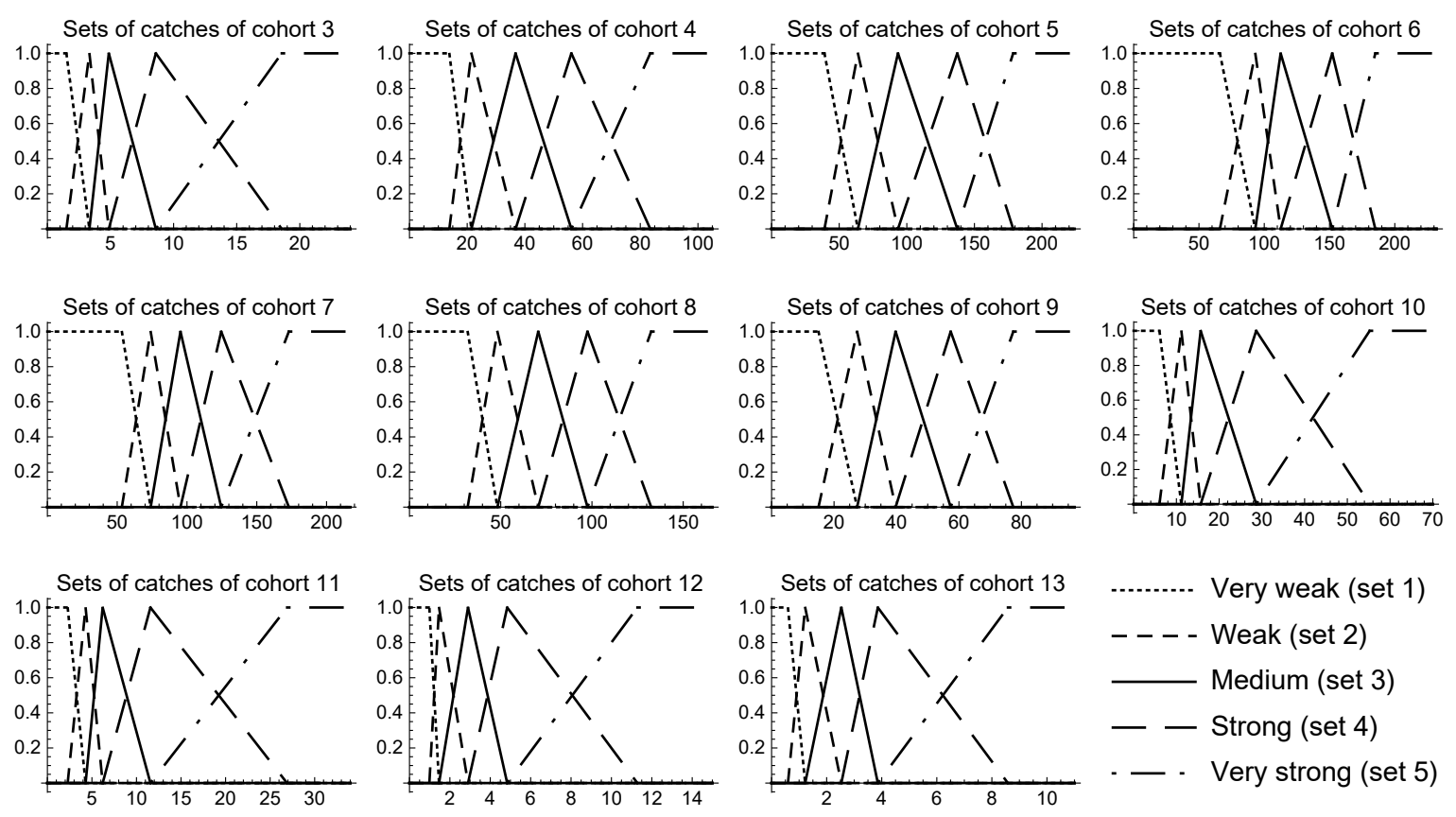

Figure 3: Membership degrees (vertical axes) for the five sets identified in Figure 2 for age groups from age 3 years to 13 years and older, as functions of catch-at-age (horizontal axes).

Table 4: Defuzzification rules employed to single out the fuzzy set of each year class by the use of sets of highest membership degrees for each age group. The numbers are referring to the sets presented in Figure 3; 1: very weak, 2: weak, 3: medium, 4: strong and 5: very strong.

\begin{tabular}{lllll}
\hline$\{1,1\} \rightarrow 1$ & $\{1,2\} \rightarrow 1$ & $\{1,3\} \rightarrow 2$ & $\{1,4\} \rightarrow 2$ & $\{1,5\} \rightarrow 3$ \\
$\{2,1\} \rightarrow 1$ & $\{2,2\} \rightarrow 2$ & $\{2,3\} \rightarrow 2$ & $\{2,4\} \rightarrow 3$ & $\{2,5\} \rightarrow 3$ \\
$\{3,1\} \rightarrow 2$ & $\{3,2\} \rightarrow 2$ & $\{3,3\} \rightarrow 3$ & $\{3,4\} \rightarrow 3$ & $\{3,5\} \rightarrow 4$ \\
$\{4,1\} \rightarrow 2$ & $\{4,2\} \rightarrow 3$ & $\{4,3\} \rightarrow 3$ & $\{4,4\} \rightarrow 4$ & $\{4,5\} \rightarrow 5$ \\
$\{5,1\} \rightarrow 2$ & $\{5,2\} \rightarrow 3$ & $\{5,3\} \rightarrow 4$ & $\{5,4\} \rightarrow 5$ & $\{5,5\} \rightarrow 5$ \\
\hline
\end{tabular}

way: $\{4,2,1,1,3,5,5\},\{5,3,1,1,2\},\{3,4,2\}$, and $\{2\}$. The final digit -2 - is assumed to represent an overall classification of the year class strength in terms of being a full member of the corresponding set, in our example this means that the 1943 year class is found to be a weak year class.

The lower row of Figure 4 displays the result after applying the second defuzzication method on all the year classes, employing the rules displayed in Table 4. The categorisation of year class strength of the period from year class 1943 to year class 2012 (recruited to the exploitable fish stock in 2015) then gives the following list for the NEA cod year class strength: $\{2,2,1,1,3,5,5,5,3,1,2,4,2,2,3,4,4,2,1,3,5,5,1,1,1,1,3,5,1,3,2$, $1,2,1,1,1,1,1,2,3,5,1,1,1,1,2,4,3,2,1,1,3,4,2,3,2,1,4,1,3,3,5,4,2,1,1,1,1,3$, 2 \}. In the graphical representation of this vector in the lower row of Figure 4 each value (year class strength) is represented by different colours. 


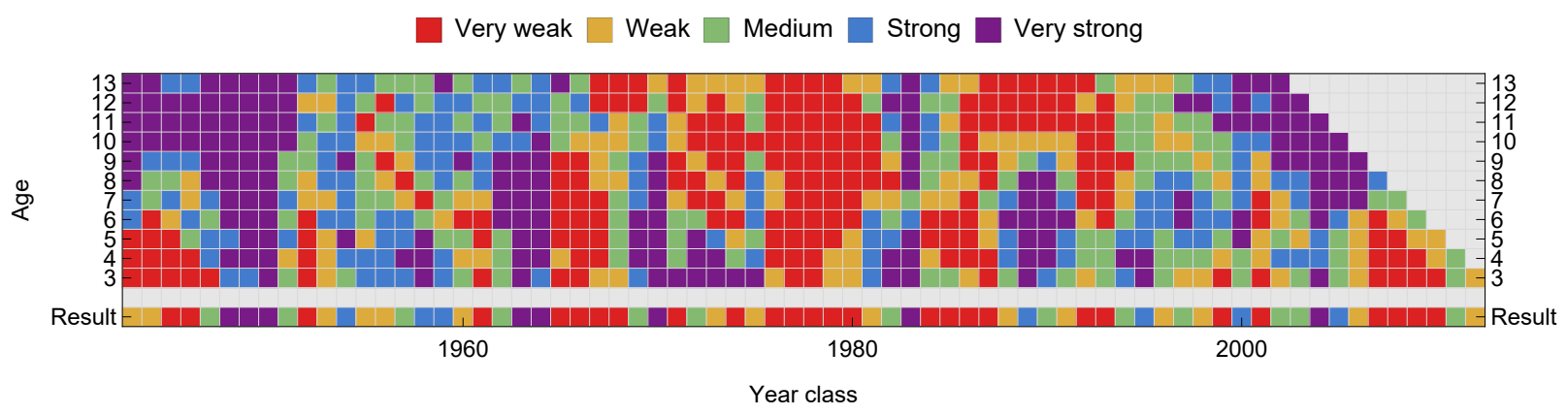

Figure 4: The top eleven rows display results after the first defuzzification procedure according to the principle of Highest Membership Degree for each year class (horizontal axis). The lower line (Result) shows the resulting aggregate after the second defuzzification procedure involving the rules displayed in Table 4.

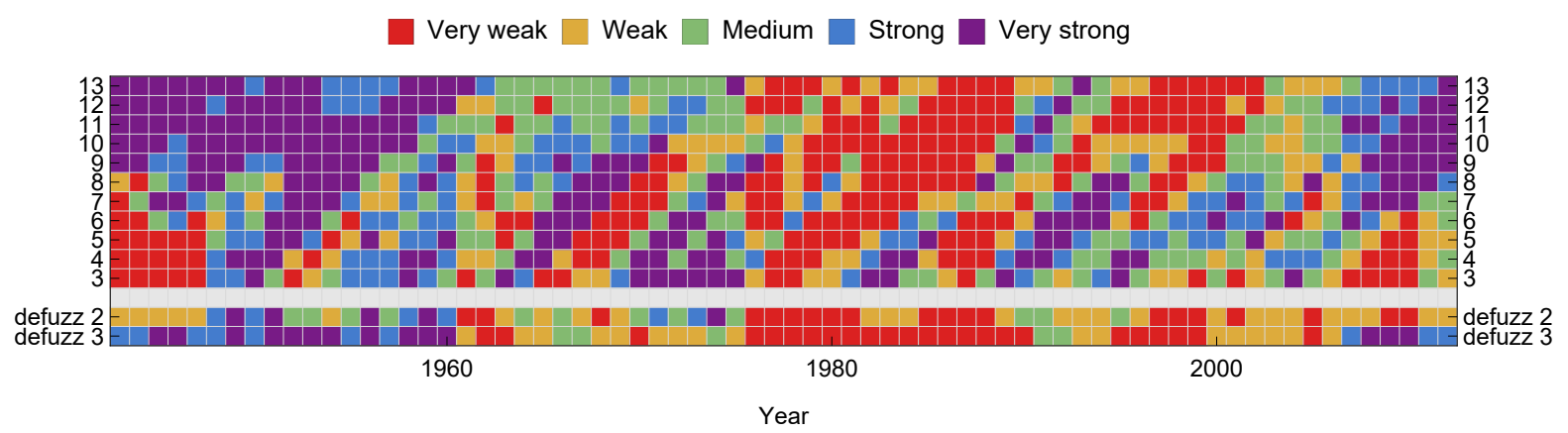

Figure 5: The top eleven rows display results after the first defuzzification procedure according to the principle of Highest Membership Degree for all year classes present in a given year (horizontal axis). The two lower lines (defuzz2 and defuzz3) show the resulting aggregates after the second and third defuzzification procedure, both employing the rules displayed in Table 4.

Up to now we have focused year class strength (e.g. recruitment strength) of each year class. Let us shift focus to now look at the assessment of relative stock size for each year, assuming this to be reflected in an aggregate of the catch-at-age evaluations of all year classes constituting the stock the given year.

The second defuzzification procedure aimed to minimise the impact from fishing by emphasising catches of young fish. A third defuzzification procedure is introduced by the opposite strategy; emphasising the catches of older fish. Previous catches on a year class will affect later catches of the same year class, and the third defuzzification procedure takes advantage of this knowledge. The procedure follows these steps: First, omitting the four youngest year classes before pairing the age groups (catch-at-age values of a given year) in the following way: (8 and 9$)$, (7 and 8), (6 and 7), (5 and 6), (4, and 5), (3 and 4), (3 and 13+), (12 and 13+) and (11 and 12), employing the rules provided in Table 4. The procedure is then repeated as in the second defuzzification procedure until only one digit characterises the strength of the exploited stock that particular year. The assumption is that the difference between the second and third defuzzification procedures 
gives a proxy of the fishing intensity. Both procedures utilise the same catch information, facing the same age composition in the stock. While the impact of fishing is believed to be low while nesting information from the younger to the older, it is assumed to be high while nesting information from the older to the younger fish.

Indicator values obtained from age distributed catches 1946 - 2015 by using the two defuzzification procedures are shown in the two lower rows of Figure 5 (note that the horizontal axis here refers to year, not year classes as in Figure 4). Since the difference between the two defuzzification procedures is assumed to reflect a fishing effort measure, we now make use of the aggregated catch data of each year to estimate a catch per unit of effort value, commonly considered to reflect stock strength (or stock density measure).

\section{Results}

The methodology described above is employing fuzzy set theory in its simplest version. The suggested defuzzification methods are illustrated by case studies to provide examples on how the theory could be implemented. Commercial catch-at-age data in the NEA cod fishery reveals a year class strength or recruitment pattern in striking correspondence with the recruitment strength estimated by stock assessments. In Figure 6 the two data sets are displayed in a common axes system. The correlation coefficient of the two data sets is $86 \%$.

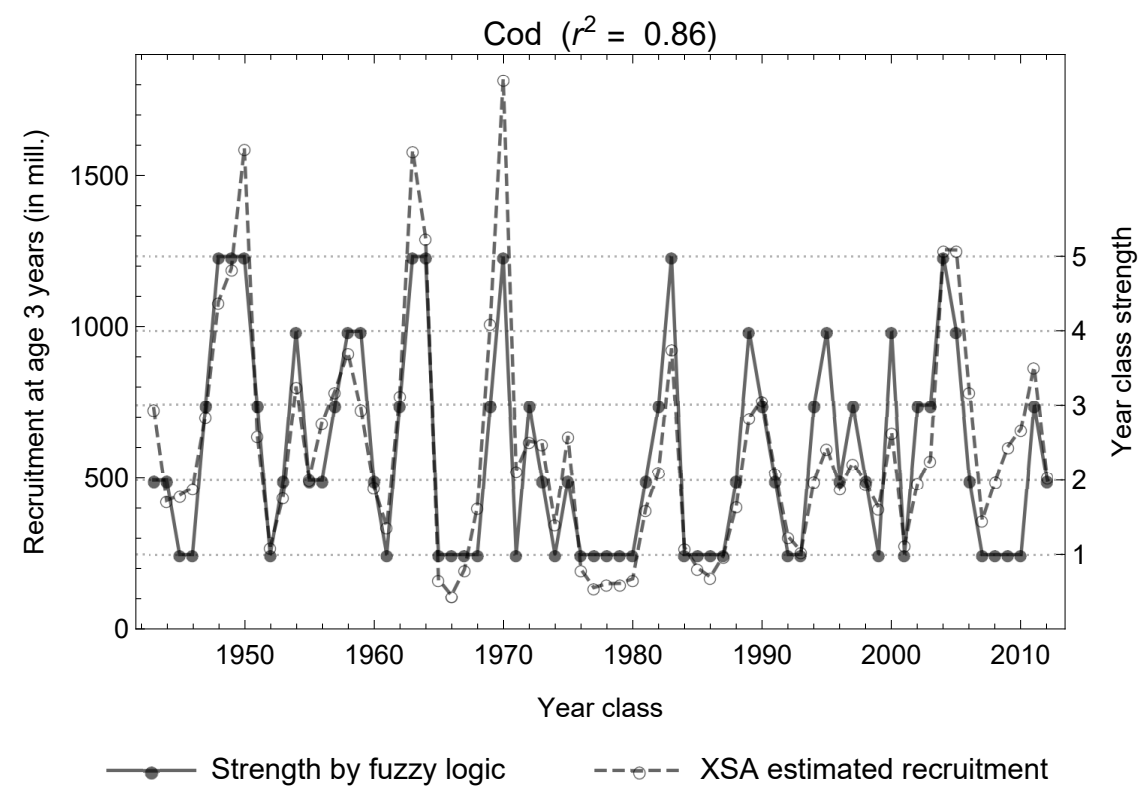

Figure 6: Results of the second defuzzification procedures as described in this paper (solid line) compared with corresponding estimates of year class strength (recruitment numbers) by stock assessments, Table 3.24a in Anon. (2016) (dashed line).

When comparing year class strengths obtained here by stock assessment estimates, a resolution of five sets (or strength categories) seems to be sufficient to grasp the essential 


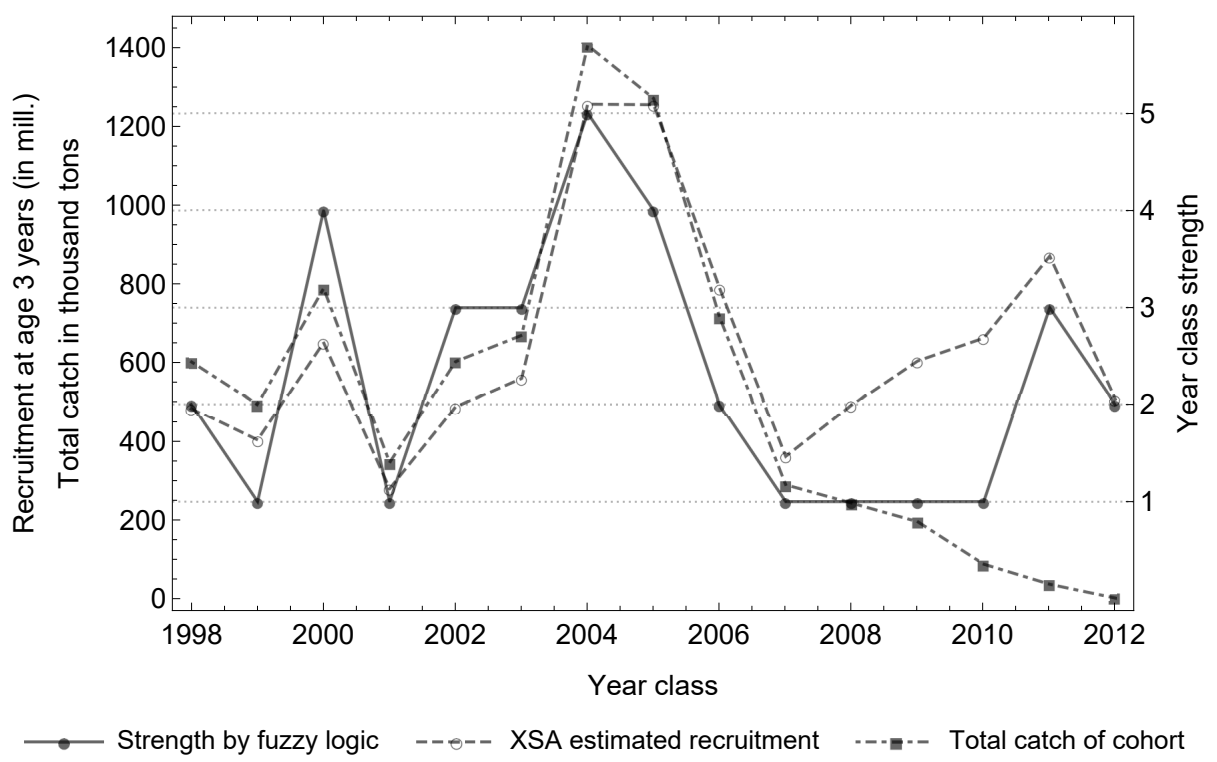

Figure 7: The figure shows the most recent (since 1998) year classes displayed in Figure 6 (solid and dashed lines) in addition to the aggregated catches of each year class (dotted line).
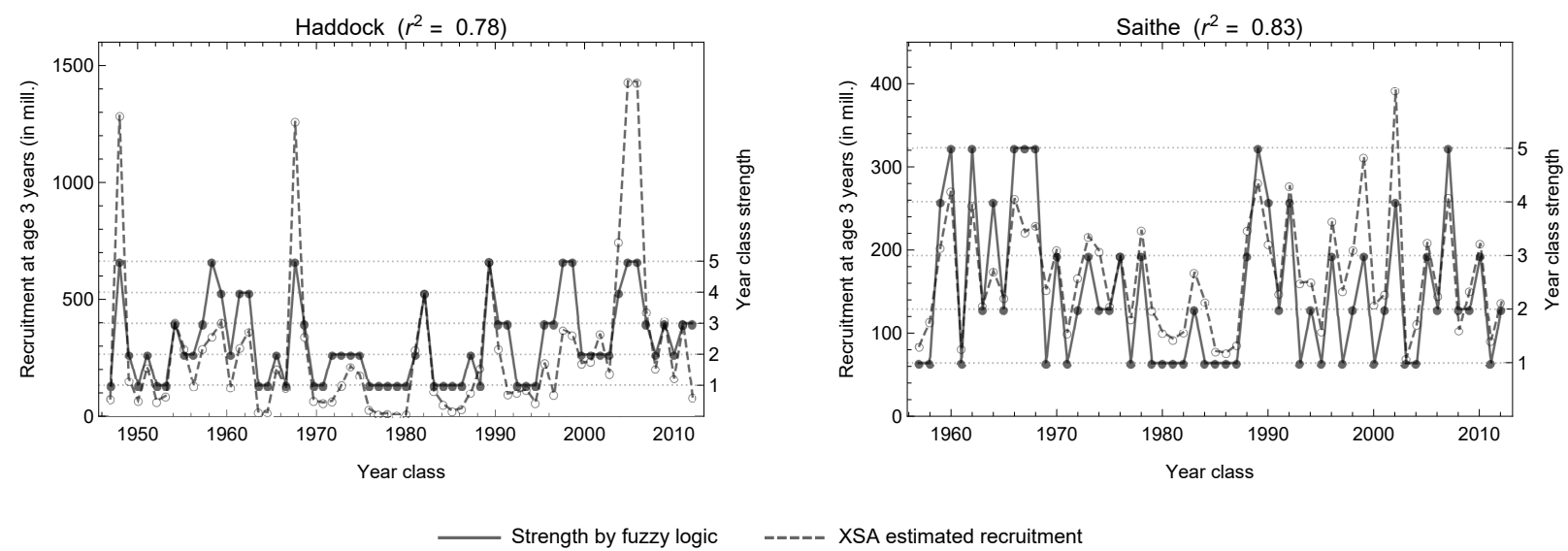

Figure 8: Results for Northeast Arctic haddock (tables 4.4 and 4.5 in Anon., 2016) and saithe (tables 5.5.1 and 5.5.2 in Anon., 2016) following the same procedure as for NEA cod in Figure 6.

fluctuations in recruitment strength over time. In the case of NEA cod, total catch by year classes correlates even stronger to the stock assessment estimates (Eide, 2016). This requires, however, a completed catch history of the year class in question; hence, it has less value for the management of a fishery. This is illustrated in Figure 7, which shows the most recent NEA cod year classes from Figure 6, in addition to total catches of the same year classes. The fuzzy logic estimated year class strengths correspond quite well to the stock assessments.

In addition to the case of the NEA cod stock, the suggested method of year class strength classification also has been applied on the catches of Northeast Arctic (NEA) haddock and saithe (Anon., 2016). Following the same procedure as described above 


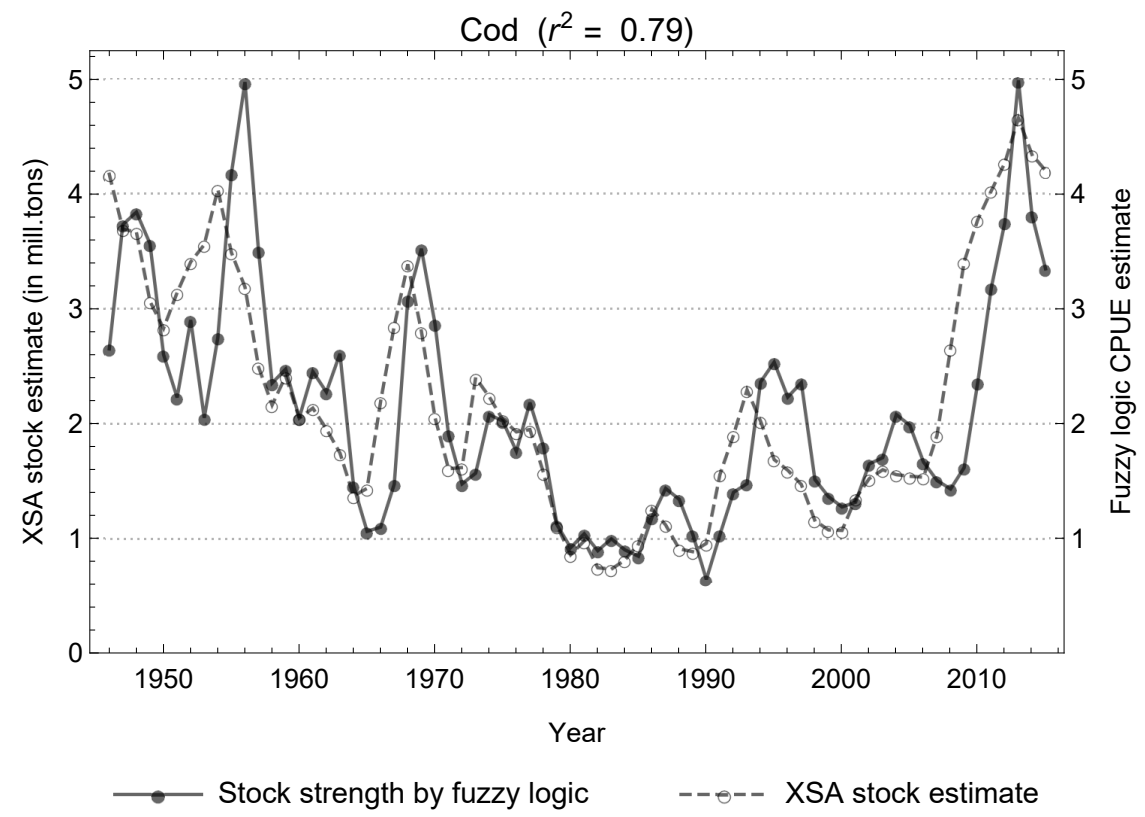

Figure 9: Total catches divided by the differences between the second and third defuzzification procedures (solid line), representing a stock size indicator (catch per unit of effort) compared with corresponding estimates of stock sizes by stock assessment 1946 - 2015, Table 3.24a in Anon. (2016) (dashed line).

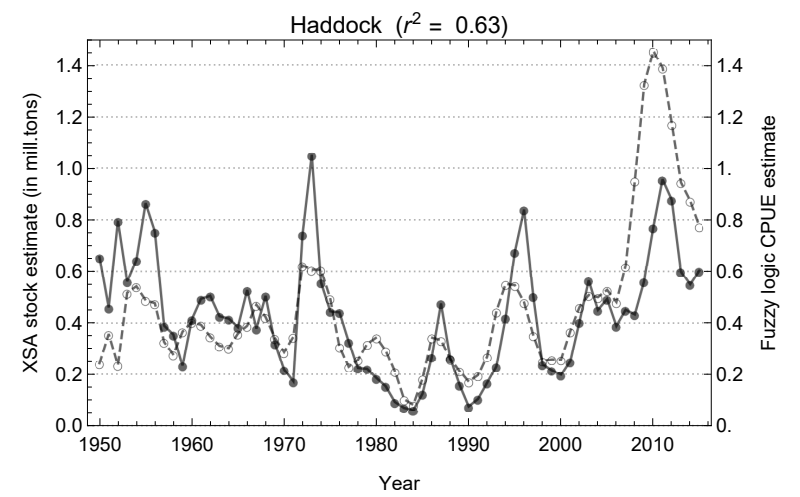

Stock strength by fuzzy logic

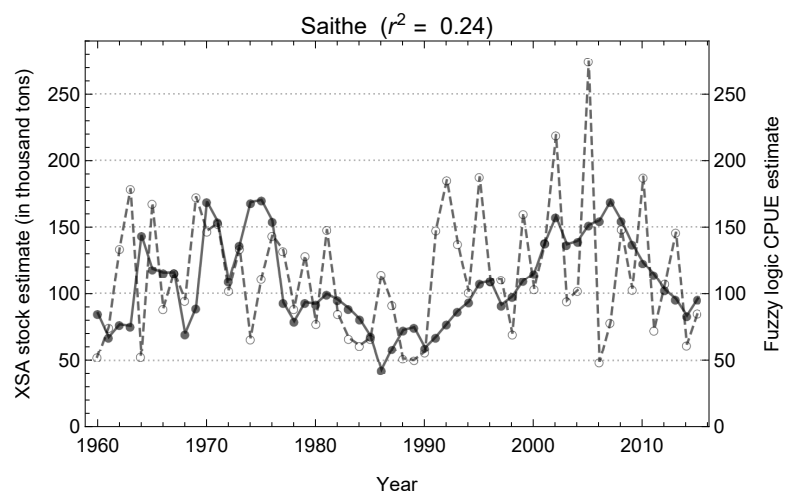

XSA stock estimate

Figure 10: Total catches divided by the differences between the second and third defuzzification procedures (solid lines), representing a stock size indicator (catch per unit of effort) compared with corresponding estimates of stock sizes by stock assessment, Table 4.17 (haddock) and Table 5.5.5 (saithe) in Anon. (2016) (dashed lines).

$78 \%$ of the stock assessment explained fluctuations in the haddock recruitment and a corresponding $83 \%$ of the saithe recruitment are explained by the methodology suggested here. The results are displayed in Figure 8.

Figure 9 shows how the sequences of recruitment strength evaluations may be developed further to a stock size indicator. The figure illustrates how a stock indicator obtained by dividing total catch with the difference between the second and third defuzzification procedures performs compared with the corresponding stock assessment estimates. $79 \%$ 
of the stock variation found by stock assessments is explained by the fuzzy logic methodology in the case of the NEA cod stock. For haddock and saithe corresponding correlation coefficients are $63 \%$ and $24 \%$ (Figure 10).

\section{Discussion}

Fuzzy logic represents a way to structure data, information and knowledge in a nonalgorithmic manner:

"Fuzzy logic provides a natural framework for the management of uncertainty in expert systems because - in contrast to traditional logical systems - its main purpose is to provide a systematic basis for representing and inferring from imprecise rather than precise knowledge. In effect, in fuzzy logic everything is allowed to be - but need not be - a matter of degree." (Zadeh, 1983)

The estimating procedures presented here are not supposed to replace stock assessment methodologies. They rather represent possible ways of developing an alternative toolbox without the strict constraints in terms mathematical formulations and increasing demand for data beyond standard catch statistics. The alternative toolbox makes use of existing knowledge (precise and imprecise) as it develops, in addition to easily available data such as commercial catch statistics. As stated in the introductory part the cost associated with data sampling is only one of the issues calling for new methods to be developed.

In order to operationalise the increased focus on possible ecosystem consequences, which modern fisheries management is committed to do, rule based methods appear to be more promising. Combined inputs (indicator values) of direct observations fed into HCRs, while taking into consideration precautionary levels corresponding to precise and imprecise knowledge about system resilience and vulnerability, seems to represent a better approach to increased complexity in the object functions of fisheries management.

Compared with corresponding findings by the use of stock assessment, the results presented here provide surprisingly consistent year class strength evaluations. The task of employing fuzzy logic methodology to estimate stock size indicators is more challenging. The results from the stock size evaluation method suggested here are promising but the methodology needs to be further developed. Such a development should also include an investigation of using catch-at-age in terms of number of individuals rather than catch weights. Using both measures may improve the overall performance of the fuzzy logic method.

The stock size indicators for the NEA cod and haddock stocks fit quite well the stock assessments while in the case of the NEA saithe stock the fuzzy logic method fails to obtain the short-term large fluctuations identified by the traditional stock assessment methods 
(Figures 9 and 10 ). The long-term trend captured by fuzzy logic is however in line with the stock assessment. A further discussion of the differences found by the two methods in the case of NEA saithe should also include a closer study of the stock assessment, in this case how the XSA methodology has been implemented. This is outside the scope of this paper but it could be noted that according to the relatively stable recruitment dynamics of the stock (Figure 8) the large fluctuations in stock size at first sight are surprising. The consistency between the two obviously is linked to the fact that the overall saithe stock size, according to stock assessments, is far below the levels of NEA cod and haddock.

From Figure 9 it seems to be a slight delay in time between the fuzzy logic method and the NEA cod stock assessment. This delay may be related to a delay between fishing effort and the impact it has on the stock. Another - or additional - explanation may be that while the stock assessment provides an evaluation of all year classes, the indicator obtained by fuzzy logic only consider the year classes represented in the fishery. A further refinement with regard of possible time lag issues may also contribute in improving the method.

This paper illustrates how fuzzy logic techniques could be used to identify stock properties commonly utilised as HCR indicators, substituting model-based indicators. Today catch data (observations) are channelled to the HCRs through non-transparent models. Heuristic expert system procedures similar to those presented in this paper could in the future be an integral part of sets of HCRs. Use of catch-at-age statistics as direct inputs to sets of HCRs will provide more explainable Lines-of-reasoning. The evaluation of the state of the stock could be one step in a rule-based line-of-reasoning in producing fisheries management decisions (or advices).

A full implementation of a fuzzy logic based heuristic expert system for the management of fisheries involves several issues not covered here. Obviously the performance of any new set of HCRs to be used in a fishery needs to be thoroughly evaluated before taken into use. Selection of indicators depends on the management objectives and how these should be embedded into the HCRs. Ideally all indicators should be measurable in order to reduce the problem of non-transparency and to be organised in the manner of a comprehensible Line-of-reasoning, containing heuristic rules based on expert knowledge and stated objectives of the fishing activity. Also non-biological indicators, such as economic performance and other social factors, could be included in the set of rules constituting the HCRs.

The simple rule-based pattern of the control system also opens for learning and then the possibility of adjusting the rules according to new knowledge gained by using the control system. A fully adaptive heuristic control system also includes the possibility of adding indicators, as long as these could be measured through observations. Such ideas also open for the possibility of developing HCRs through sets of meta-rules, a control system which in principle constitute a system of artificial intelligence (AI) with 
the capacity of learning.

The aim of this paper has been to investigate the ability of simple, intuitive rules, providing fisheries managers with essential information about the state of the stock. The results are promising even though there may be a long way to go until such ideas can make their way into the fairly well-functioning management procedures of modern fisheries today. Perhaps the first practical use of fully developed rule-based expert systems rather will be in less data-rich fisheries where the management procedures today are poorly developed.

\section{Acknowledgments}

The research leading to these results has received funding from the European Unions Horizon 2020 research and innovation programme under grant agreement no 727891. The author also wishes to thank the editor and two reviewers for valuable comments.

\section{References}

Anon. (1996). Precautionary approach to capture fisheries and species introductions. FAO Fisheries Technical Paper, No. 350, Part 2, FAO, Rome.

Anon. (2006). Report of the Study Group on Management Strategies (SGMAS). ICES Document. ICES CM 2006/ACFM:15, 157pp.

Anon. (2016). Report of the Arctic fisheries working group (AFWG). ICES Advisory Committee. ICES CM 2016/ACOM:06. Copenhagen, Denmark, pp. 622.

Clancey, W. J. (1983). The Epistemology of a Rule-Based Expert System - a Framework for Explanation. Artificial Intelligence, 20(3), 215251. https://doi.org/10.1016/00043702(83)90008-5

Dankel, D. J., Vlstad, J. H., Aanes, S., and Marshall, C. T. (2016). Communicating uncertainty in quota advice: a case for confidence interval harvest control rules (CIHCRs) for fisheries 1. Canadian Journal of Fisheries and Aquatic Sciences, 73, 3093017. https://doi.org/10.1139/cjfas-2015-0078

Degnbol, Paul (2001). Science and the user perspective The scale gap and the need for shared indicators. Paper presented at People and the Sea Inaugural Conference Center for Maritime Research (MARE), Amsterdam, The Netherlands. http://www.ifm.dk/reports/60.pdf

Eide, A. (2016). Management performance indicators based on year-class histories. Fisheries Research, 174, 280-287. https://doi.org/10.1016/j.fishres.2015.10.026 
Feigenbaum, Edward A. (1977). The Art of Artificial Intelligence. 1. Themes and Case Studies of Knowledge Engineering. Working paper, Stanford Heuristic Programming Project, Memo HPP-77-25, Stanford University. 18 pp. http://www.dtic.mil/docs/citations/ADA046289

Garcia, Serge Michel, Rey-Valette, Hlne, and Bodiguel, Clotilde (2009). Which Indicators for What Management? The Challenge of Connecting Offer and Demand of Indicators. In Cochrane, K. L., and Garcia, S. M. (2009). A fishery managers guidebook. Published by The Food and Agriculture Organization of the United Nations and Blackwell Publishing. ISBN: 978-92-5-105963-0. Chapter 12, 303-335. http://www.fao.org/docrep/015/i0053e/i0053e.pdf

Gulland, J. A. (1965). Estimation of mortality rates. Annex to Arctic Fisheries Working Group Report. ICES CM 1965. Vol.3.

Kelly, C. J., and Codling, E. A. (2006). Cheap and dirty fisheries science and management in the North Atlantic. Fisheries Research. https://doi.org/10.1016/j.fishres.2006.03.007

Kvamsdal, S. F., Eide, A., Ekerhovd, N.-A., Enberg, K., Gudmundsdottir, A., Hoel, A. H., Mills, K. E., Mueter, F. J., Ravn-Jonsen, L., Sandal, L. K., Stiansen, J.E, and Vestergaard, N. (2016). Harvest control rules in modern fisheries management. Elementa: Science of the Anthropocene, 4(1), 114. https://doi.org/10.12952/journal.elementa.000114

Mackinson, S., Vasconcellos, M., and Newlands, N. (1999). A new approach to the analysis of stock-recruitment relationships: model-free estimation using fuzzy logic. Canadian Journal of Fisheries and Aquatic Sciences, 56(4), 686-699.

Punt, A. E. (2006) The FAO Precautionary Approach after almost 10 years: have we progressed towards implementing simulation-tested feedback-control management systems for fisheries management? Natural Resource Modeling 19:441-464.

Sakuramoto, K. (1995). A Method to Estimate Relative Recruitment from Catch-at-Age Data Using Fuzzy Control Theory. Fisheries Science, 61(3), 401-405.

Schnute, J. T. and L. J. Richards (2001). Use and abuse of fishery models. Can. J. Fish. Aquat. Sci. 58:10-17.

Shepherd, J. G. (1999). Extended survivors analysis: An improved method for the analysis of catch-at-age data and abundance indices. ICES Journal of Marine Science, 56(5), 584-591. https://doi.org/10.1006/jmsc.1999.0498 
Silvert, W. (1997). Ecological impact classification with fuzzy sets. Ecological Modelling, 96(1-3), 1-10. Retrieved from http://www.sciencedirect.com/science/article/pii/S0304380096000518

Zadeh, L. A. (1983). The role of fuzzy logic in the management of uncertainty in expert systems. Fuzzy Sets and Systems, 11(13), 199227. https://doi.org/10.1016/S01650114(83)80081-5.

Zadeh, L. A. (2002). Toward a perception-based theory of probabilistic reasoning with imprecise probabilities. Journal of Statistical Planning and Inference, 105(1), 233-264. 